\title{
Performance-enhanced NOMA-VLC using subcarrier pairwise coding
}

\author{
Jin $\mathrm{Shi}^{1}$, Jing $\mathrm{He}^{1 *}$, Yang Hong ${ }^{2,3}$, Jing $\mathrm{He}^{1}$, and Lian-Kuan Chen ${ }^{2}$
}

1. College of Computer Science and Electronic Engineering, Hunan University, Changsha 410082, China

2. Department of Information Engineering, The Chinese University of Hong Kong, Hong Kong SAR, China

3. Currently with Optoelectronics Research Centre, University of Southampton, Southampton SO17 1BJ, United Kingdom

\begin{abstract}
To alleviate the error propagation issue of non-orthogonal multiple access (NOMA) based visible light communication (VLC) systems, we propose and experimentally demonstrate the subcarrier pairwise coding (PWC) based NOMA-VLC. Without extra coding overhead, the computation-efficient subcarrier PWC can significantly improve the overall system performance by pairing the subcarriers of high signal-to-noise ratios (SNRs) with those of low SNRs. Under different transmitted baud rate from $150 \mathrm{MSa} / \mathrm{s}$ to $225 \mathrm{MSa} / \mathrm{s}$, the bit error rate (BER) performance with and without PWC is investigated in a NOMA-VLC system. Experimental results show that with the optimized power ratio between the NOMA users, up to 3.3-dB $\mathrm{Q}^{2}$-factor improvement can be achieved by the proposed scheme.
\end{abstract}

Keywords: Visible light communications (VLC); non-orthogonal multiple access (NOMA); optical communications; pairwise coding.

\section{Introduction}

Visible light communication (VLC) is a promising supplement to the conventional radio frequency (RF) communication for future high-speed wireless access and has attracted increasing interests from both academia and industry [1], [2]. Compared to the RF technology, VLC exhibits advantages such as license-free, lower cost and higher security [3]. However, the main bottleneck of VLC is the limited modulation bandwidth, which severely limits the achievable capacity of VLC systems. In order to enhance the aggregate capacity, as well as to provide the capability of multiple access for multiple users, the conventional multiple access technologies, such as frequency division multiple access (FDMA), time division multiple access (TDMA) and code division multiple access (CDMA) have been employed in VLC [4], [5]. Apart from the aforementioned multiple access technologies, orthogonal frequency division multiple access (OFDMA) has been widely investigated in optical communication systems due to better ability to resist the frequency selectivity channel and inter-symbol interference (ISI) [6-8]. It has been demonstrated that orthogonal frequency division multiple access (OFDMA) can be an effective solution for VLC to realize high system throughout and to mitigate the multi-path interference, thus it has been widely investigated in VLC systems [9]-[11]. Compared to OFDMA, non-orthogonal multiple access (NOMA) exploits the new approach of user multiplexing in the power-domain [12]. NOMA provides higher spectral efficiency than the conventional schemes, as it superposes multiple users' signals in the power domain while sharing the same time-frequency resources [13]. Moreover, by allocating more power to the user with poor channel condition, NOMA can effectively balance the trade-off between user fairness and system throughout [14]. It has also been proven that OFDM-based NOMA exhibits higher system capacity and better user fairness in VLC [15], [16].

For NOMA-based systems, successive interference cancellation (SIC) algorithm is commonly used to separate the overlapped signals at the receiver [14], [16]. However, the performance of the SIC algorithm is limited by its inherent error propagation issue. For example, the user with strong power allocation (also referred to as the strong user, UE1) will be first decoded. Then, prior to the decoding of the user with weak power allocation, which is referred to as the weak user (UE2), SIC is performed to subtract the reconstructed strong user's signal component from the overlapped signal, so as to eliminate the superposing interference. 
Consequently, if the strong user is not correctly decoded, the resulting error will propagate to the subsequent weak user, thus degrading the system performance. The error propagation issue is particularly severe for VLC since it generally suffers from the high-frequency fading [17]. Some works focusing on eliminating the error propagation have been investigated in NOMA-VLC system [18, 19]. In [18], symmetric superposition coding (SSC) and symmetric SIC decoding are proposed in downlink NOMA system, which can eliminate 90\% demodulation errors caused by error propagation. In [19], a novel NOMA scheme based on constellation partitioning coding (CPC) and uneven constellation de-mapping (UCD) for downlink VLC is proposed. The user decoding can be realized without SIC, hence no error propagation issue arises. Compared with the traditional NOMA, these schemes can improve the system performance of users requiring SIC operation. However, they have no performance enhancement for UE1. The frequency roll-off imposes inevitable performance degradation at high frequencies, which results in worse system performance for NOMA-based VLC. Pairwise coding (PWC) is an effective scheme to improve system performance. While requiring no overhead and exhibiting low computation complexity, PWC can pair two different components that have imbalanced channel conditions to achieve better system performance [20-22]. In [21], PWC is introduced to the layered/enhanced asymmetrically clipped optical OFDM (L/EACO-OFDM) to mitigate the distortion from lower layer to higher layers. In [22], in order to further enhance the system capacity, subcarrier PWC combined with adaptive bit-loading is proposed for VLC.

To resolve the issue of high-frequency fading induced error propagation in NOMA-based VLC, in this paper, we propose and experimentally demonstrate the pairwise coding (PWC) based OFDM-NOMA VLC. The key idea of the proposed scheme is to address the high-frequency fading issue in the NOMA VLC system. By resolving this severe issue with the proposed scheme, both users can achieve better BER performance. The improvement of the weak user is from two folds. One is the direct improvement from the PWC. The other one is indirect improvement as the error propagation from the strong user to the weak user is significantly mitigated, since the BER performance of the strong user itself has been improved. The performance improvement of PWC is attributed to the maximized signal space diversity by rotating the constellation, which is achieved by exchanging the real and imaginary components between different sub-channels. For the proposed PWC-based OFDM-NOMA VLC, the low-frequency subcarriers that have better signal-to-noise ratio (SNR) conditions are paired with the high-frequency subcarriers (with worse SNRs). As a result, each pair of subcarriers can achieve similar transmission performance, and the high-frequency fading issue is effectively resolved. The error propagation can be effectively mitigated due to the reduced error bits in UE1. We show that for $225 \mathrm{MS} / \mathrm{s}$, by using the proposed scheme, the $\mathrm{Q}^{2}$-factor $\left(\mathrm{Q}^{2}(\mathrm{~dB})=20 \log 10\left(\sqrt{e r f c^{-1}(2 \times B E R)}\right)\right)$ can be improved from $6.5 \mathrm{~dB}$ to $7.6 \mathrm{~dB}$ and 4.4 to 6.6 for UE1 and $\mathrm{UE2}$, respectively, resulting in an aggregate $\mathrm{Q}^{2}$-factor improvement of $3.3 \mathrm{~dB}$.

\section{Principle}

The schematic diagram of PWC-based OFDM-NOMA VLC is depicted in Fig. 1. We assume that NOMA signal serves two users at the transmitter (TX). Fig. 1(a) shows the process diagram of NOMA modulation with two users. Subcarrier PWC is applied for both UE1 and UE2 and then both users are modulated using OFDM signal. Note that to generate real-valued and non-negative OFDM signal for intensity-modulation/direct-detection (IM/DD) optical systems, e.g., VLC systems, Hermitian symmetry is adopted to obtain the real-valued OFDM signal. The resulting signal, together with a direct-current (DC) bias, can then be used to drive a visible light laser or light-emitting diode (LED) in VLC. Power factors are used to control the power of users. After multiplying the signal of each user by the corresponding power factor, the signals of the two users are superposed in power domain and the overlapped signal is represented by $X=\sqrt{P_{S}} S+\sqrt{P_{W}} W$, where $S$ denotes UE1's information that has a strong power of $P_{S}$, and $W$ denotes UE2's information that has a weak power $P_{W}$. Note that $P_{S}$ is always larger than $P_{W}$ as illustrated in Fig. $1(\mathrm{~d})$. At the 
receiver, after signal equalization and subcarrier pairwise decoding, UE1 with a lager power is firstly decoded by treating UE2's signal as interference. Prior to the decoding of UE2, SIC algorithm is utilized to
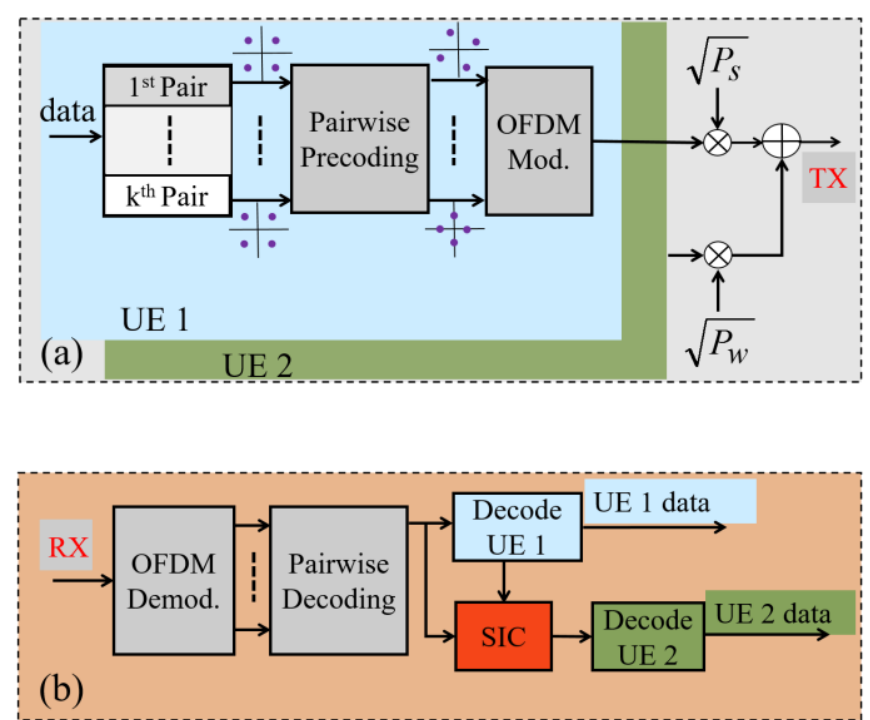
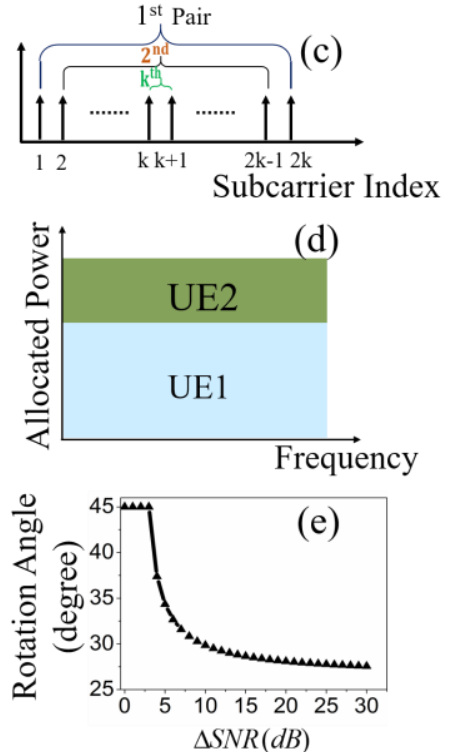

Fig. 1. The schematic diagram of PWC-based OFDM-NOMA VLC. (a) NOMA modulation with pairwise precoding, (b) NOMA demodulation with pairwise decoding, (c) subcarrier pairing scheme for each user, (d) user allocation in power domain, (e) optimal rotation angle versus $\triangle S N R$ for $4 \mathrm{QAM}$ modulation.

recover $W$ by subtracting the signal of UE1 from the overlapped signal. As a result, the reconstructed signal $W$ can be described as $W=\left(X-\sqrt{P_{S}} * S\right) / \sqrt{P_{\mathrm{w}}}$. The diagram of demodulation processing of PWC based OFDM-NOMA VLC is illustrated in Fig. 1(b).

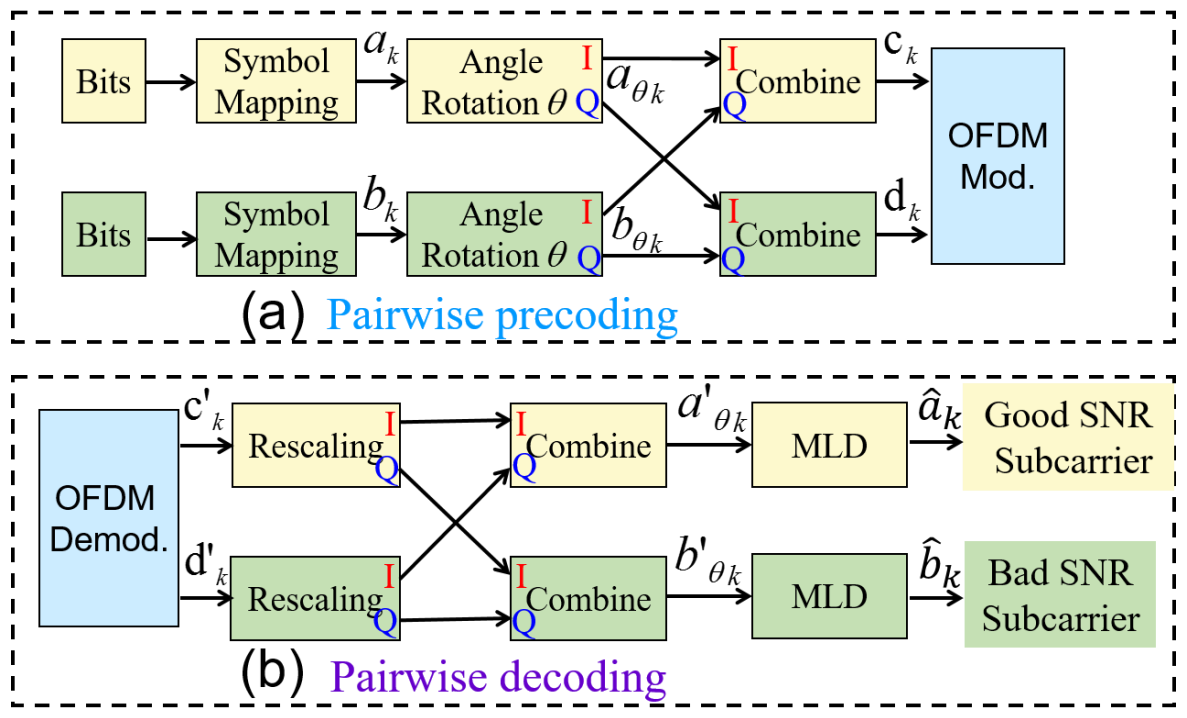

Fig. 2. Subcarrier pairwise (a) precoding and (b) decoding for one pair of subcarriers.

The flow chart diagram of the PWC for a pair of subcarriers is shown in Fig. 2. At each user, subcarrier pairwise precoding is performed before OFDM modulation. The idea of subcarrier PWC is that by pairing the unequal-SNR subcarriers, the power of I and Q components are not identical. Then, rotating the constellation can maximize the signal space diversity, leading to performance improvement. Assuming there are $2 \times k$ subcarriers, we take $k$ pairs of subcarriers consisting of good SNR subcarriers and bad SNR subcarriers. For example, as shown in Fig. 1(c), the $1^{\text {st }}$ pair subcarriers contain the subcarrier index 1 and $2 \times k$ while the $k^{\text {th }}$ pair subcarriers contain the subcarrier index $k$ and $k+1$. The bit data streams are first mapped into complex 
data $a_{k}$ and $b_{k}$. Then, each pair of subcarriers is rotated by $\theta_{k}$ to attain the optimal system performance. For 4-QAM, the optimal rotation angle considering the range $[0, \pi / 4]$ is calculated as in the following equation [20]:

$$
\theta_{\text {opt }}=\left\{\begin{array}{cc}
\pi / 4 & \Delta S N R \leq 3 \\
\tan ^{-1}\left[(\Delta S N R-1)-\sqrt{(\Delta S N R)-1)^{2}-\Delta S N R}\right] & \Delta S N R>3
\end{array}\right.
$$

Apparently, the optimal rotation angle $\theta_{\text {opt }}$ for each pair of subcarriers depends on the SNR difference between each pair of subcarriers, which is denoted as $\triangle S N R$. The optimal rotation angle as a function of $\triangle S N R$ is depicted in Fig. 1(e). It can be seen that with the increase of $\triangle S N R$, the optimal rotation angle will decrease accordingly. Following the angle rotation, I/Q components interleaving is performed for each pair of subcarriers, resulting in a new pair of complex symbols as follows:

$$
\begin{aligned}
& c_{k}=\mathfrak{R}\left(a_{k} e^{j \theta_{k}}\right)+\mathfrak{R}\left(b_{k} e^{j \theta_{k}}\right) j \\
& d_{k}=\mathfrak{I}\left(a_{k} e^{j \theta_{k}}\right)+\mathfrak{I}\left(b_{k} e^{j \theta_{k}}\right) j
\end{aligned}
$$

where $a_{k}$ and $b_{k}$ are the $k^{\text {th }}$-pair of original symbols. $\mathfrak{R}(\cdot)$ and $\mathfrak{I}(\cdot)$ are the real and imaginary parts of the original symbol with an angle rotation. At the receiver, after signal equalization, the pairwise decoding is carried out, which is illustrated in Fig. 2 (b). In order to balance the noise variance on different subcarriers, each subcarrier signal is rescaled accordingly by multiplying the estimated subcarrier SNR. Then, the I/Q components from each pair of subcarriers are de-interleaved. The resulting signal can be given as:

$$
\begin{aligned}
& a_{\theta k}^{\prime}=\mathfrak{R}\left(c_{k}^{\prime} \sqrt{S N R_{\text {good }}}\right)+\mathfrak{R}\left(d_{k}^{\prime} \sqrt{S N R_{\text {bad }}}\right) j \\
& b_{\theta k}^{\prime}=\mathfrak{I}\left(c_{k}^{\prime} \sqrt{S N R_{\text {good }}}\right)+\mathfrak{I}\left(d_{k}^{\prime} \sqrt{S N R_{\text {bad }}}\right) j
\end{aligned}
$$

where $c^{\prime}{ }_{k}$ and $d_{k}^{\prime}$ are the $k^{\text {th }}$-pair symbols after channel equalization at the receiver. $S N R_{\text {good }}$ denotes the subcarrier of $k^{\text {th }}$-pair with high SNR and $S N R_{b a d}$ is the subcarrier of $k^{\text {th }}$-pair with low SNR. Finally, maximum likelihood detection (MLD) is utilized to recover the transmitted signal:

$$
\begin{aligned}
& \hat{a}_{k}=\arg \min _{c_{k}}\left\{\left|a_{\theta k}^{\prime}-r_{k}\right|^{2}\right\}, \quad \hat{b}_{k}=\arg \min _{c_{k}}\left\{\left|b_{\theta k}^{\prime}-r_{k}\right|^{2}\right\}, \\
& r_{k}=\mathfrak{R}\left(X_{k} e^{j \theta_{k}}\right) \sqrt{S N R_{\text {good }}}+\mathfrak{R}\left(X_{k} e^{j \theta_{k}}\right) \sqrt{S N R_{\text {bad }}} j
\end{aligned}
$$

where $r_{\mathrm{k}}$ is the rotated and scaled referencing points from QPSK constellation points, i.e., $X_{k}=\{1+1 \mathrm{j}, 1-1 \mathrm{j}$, $-1+1 \mathrm{j},-1-1 \mathrm{j}\}$.

To clearly illustrate the PWC processing at the transmitter, the constellations at each step of the PWC-based OFDM-NOMA scheme are given in Fig. 3, wherein the $8^{\text {th }}$ pair of subcarriers is taken as an example. Firstly, conventional QPSK constellation points with different power factors for both users are generated. Then, after angle rotation and I/Q interleaving, the subcarriers are a 16QAM-like constellation. It is worth noting that I/Q interleaving is performed between the same pair of subcarriers rather than between users. The shape of constellation after I/Q interleaving depends on the angle of rotation. After user overlapping in the power domain, an irregularly shaped constellation is attained. It is worth noting that for the PWC-based OFDM-NOMA signal, the shape of constellation not only depends on the angle of rotation but also is related to the power ratio between users.

It is worth noting that some computational complexity is sacrificed by using the PWC scheme for BER performance improvement. The extra computational complexity is analyzed as follows. In the pairwise precoding step, the angle rotation requires extra complex multiplications, and the corresponding complexity is $O\left((2 * k)^{2}\right)$. The I/Q components interleaving can be easily implemented by hardware, thus its computational complexity can be neglected. In the pairwise decoding step, each subcarrier requires rescaling accordingly by multiplying the estimated subcarrier SNR. Its complexity is $O\left((2 * k)^{2}\right)$. Similarly, the computational complexity of I/Q components de-interleaving can also be neglected as explained above. Then for MLD 
operation, the corresponding complexity is $O\left(2 * \mathrm{k}+(2 * k)^{2}\right)$ and $2 * \mathrm{k}$ operations of the argument of the minimum ( $\arg \min [\bullet]$ ). In summary, total extra computational complexity arose from the adoption of the subcarrier PWC scheme is $O\left(12 * k^{2}+2 * k\right)$ and $2 * \mathrm{k}$ operations of the argument of the minimum $(\arg \min [\cdot])$.

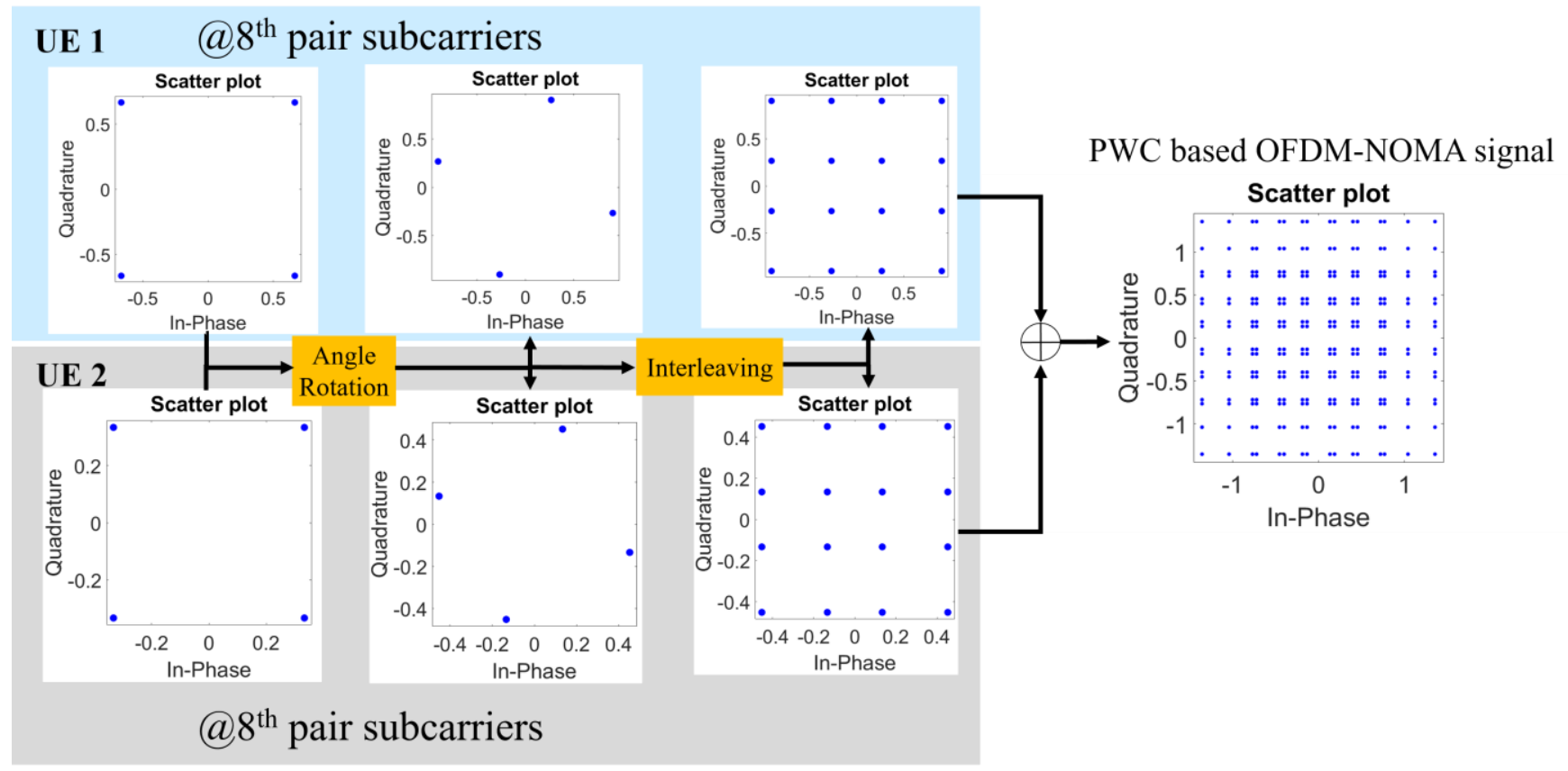

Fig. 3. The processing diagram at transmitter via constellations using $8^{\text {th }}$ pair of subcarriers as an example.

\section{Experimental Setup}

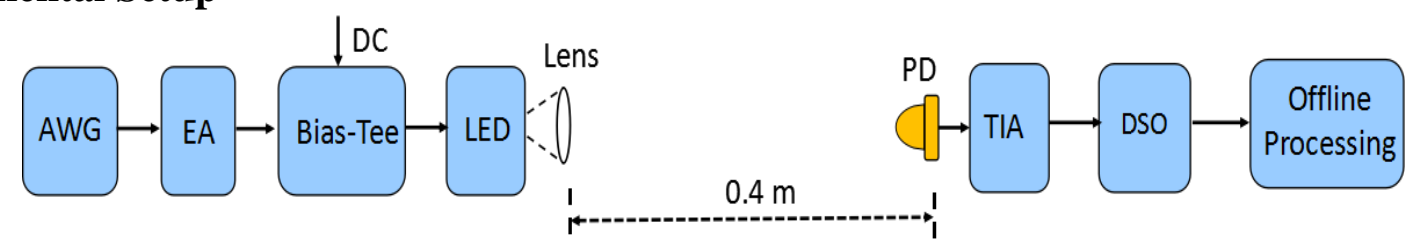

Fig. 4. Experimental setup of PWC-based OFDM-NOMA downlink VLC system.

Fig. 4 shows the experimental setup of the PWC-based OFDM-NOMA downlink VLC system. Each user employs the OFDM format. The original data bits are first mapped into complex symbols with 4-QAM. The block size of fast Fourier transform (FFT) is 256 and the length of cyclic prefix (CP) is 16. As aforementioned, Hermitian symmetry is imposed in the frequency domain to obtain real-valued signals. Therefore, only half of the subcarriers can be used to carry data, while the rest half of subcarriers are used for Hermitian symmetry. The direct-current with subcarrier index of 0 , Nyquist subcarrier (subcarrier index is 128) and other 23 positive-frequency subcarriers (subcarrier index from 1 to 6 and 111 to 127) are set to zeros due to their poor SNRs. Therefore, each OFDM symbol carries 104 data subcarriers (subcarrier index from 7 to 110) in the PWC-based OFDM-NOMA transmissions. The data subcarriers are divided into 52 pairs according to the estimated SNR from the error vector magnitude (EVM) measurement. At the transmitter, before the inverse FFT (IFFT) operation, all pairs of subcarriers are applied with subcarrier PWC. Then, power allocation is performed for each user by multiplying the power factor $P_{s}$ and $P_{w}\left(P_{s}>P_{w}, P_{s}+P_{w}=1\right)$. Subsequently, power domain multiplexing between UE1 and UE2 is performed, and the resulting NOMA signal is fed into an arbitrary waveform generation (AWG) (Tektronix 7122C) for analog signal generation. An electrical amplifier (EA) (ZHL-6A-S+) is utilized to amplify the analog signal, and the amplified signal together with a direct current (DC) bias $(3.1 \mathrm{~V})$ is then used to drive a blue LED. To increase the received light intensity, a lens is placed in front of the LED. After $0.4-\mathrm{m}$ free space transmission, the received signal is detected by a photodiode (Hamamatsu S10784). A self-designed trans-impedance amplifier (TIA) is utilized 
to amplify the detected signal. The amplified signal is then recorded by a real-time digital storage oscilloscope (DSO) (Tektronix TDS6804B) for further offline digital signal processing (DSP).

\section{Experimental results and discussions}

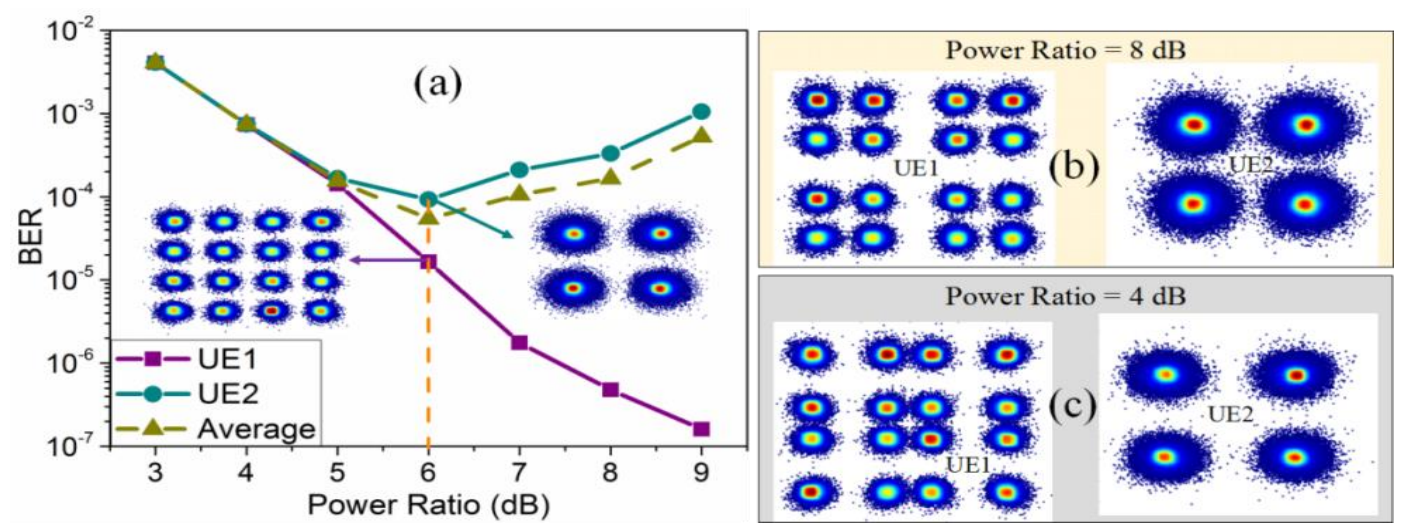

Fig. 5 (a) BER versus the power ratio between UE1 and UE2, (b) at a power ratio of $8 \mathrm{~dB}$, and (c) at a power ratio of $4 \mathrm{~dB}$.

Apparently, the power ratio between UE1 and UE2 will significantly affect the system performance of the OFDM-NOMA VLC system. Therefore, we first investigate the optimal power ratio between UE1 and UE2 without PWC. Fig. 5(a) shows the BER versus the power ratio at the transmitted baud rate of $150 \mathrm{MSa} / \mathrm{s}$. It can be seen that with the increase of power ratio, UE1 achieves better BER performance, owing to the increased signal power. In comparison, UE2 has worse BER performance, due to less allocated signal power. With a power ratio of $8 \mathrm{~dB}$, as shown in Fig. 5(b), the constellation of UE1 is obviously clearer, whereas the constellation of UE2 is more susceptible to noise owing to the reduced signal power. In contrast, when the power ratio decreases, i.e., more power is gradually allocated to UE2, it is difficult to demodulate UE1's information from the superposed signal owing to the reduced power of UE1. The deterioration of UE1 performance contributes to UE2's performance degradation as well, which is attributed to the error propagation from UE1 to UE2. Consequently, UE2 obtains the optimal BER performance when the power ratio lies in the medium level. Fig. 5(c) illustrates the constellations of UE1 and UE2, at a power ratio of $4 \mathrm{~dB}$. In order to evaluate the overall performance of the two-user NOMA-VLC system, the average BER of UE1 and UE2 is taken as the metric in this work. From Fig. 5(a), it can be found that the optimal power ratio is 6 $\mathrm{dB}$.

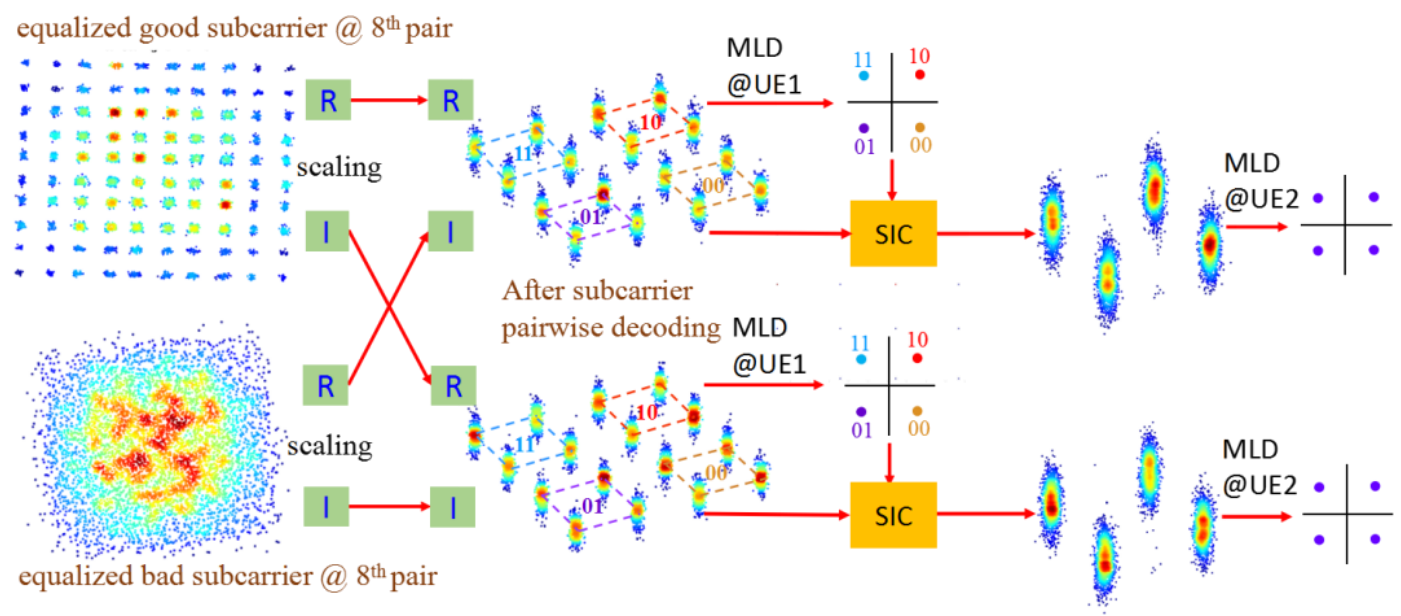

Fig. 6. The decoding process of a pair of subcarriers with PWC after signal equalization.

With the optimal power ratio, the decoding process of PWC-based OFDM-NOMA VLC is investigated. Without loss of generality, the $8^{\text {th }}$ pair of subcarriers (subcarrier index 14 as good subcarrier and subcarrier index 103 as bad subcarrier) is taken as an example, and the corresponding decoding process is shown in Fig. 
6. After signal equalization, the good subcarrier exhibits a clear irregularly-shaped constellation due to the power overlapping and I/Q interleaving, whereas the constellation located in bad subcarrier is not distinguishable due to the extremely poor SNR condition. In order to make the constellations have a similar size, the NOMA signal with subcarrier PWC is first scaled by multiplying the estimated subcarrier SNR. Then I/Q interleaving is performed to obtain the rotated 16-QAM-like NOMA signal. Finally, MLD is performed to recover the users' information.
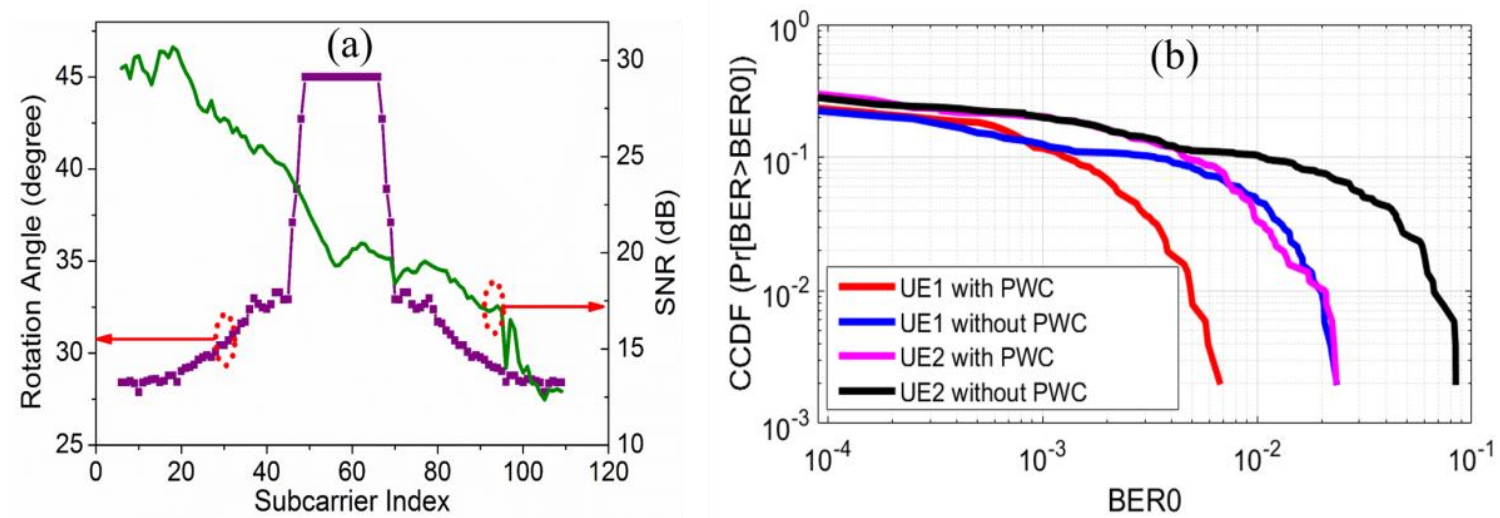

Fig. 7. (a) The rotation angle and the SNR versus subcarrier index, (b) the CCDF curve of BER distribution for NOMA-VLC system at the baud rate of $175 \mathrm{MSa} / \mathrm{s}$.

Fig. 7(a) illustrates the rotation angle and the SNR as a function of subcarrier index for the NOMA-VLC system at a baud rate of $175 \mathrm{MSa} / \mathrm{s}$. It can be seen that the high-frequency fading is particularly severe for VLC and the SNR remarkably decreases with the increase of subcarrier index. To mitigate the fading induced performance degradation, the subcarriers are divided into two groups for PWC. The corresponding rotation angle profile is given in Fig. 7(a) as well. Note that the rotation angle profile is symmetrical since the angles for subcarriers belonging to the same pair are the identical.

To evaluate the BER performance improvement on different subcarriers by using the PWC-based OFDM-NOMA, we further investigate the BER performance of all the data subcarriers. The probability of BER exceeding a certain threshold BER0 is characterized by the complementary cumulative distribution function (CCDF) for all the subcarriers, as depicted in Fig. 7(b). It can be seen that for both UE1 and UE2, the PWC scheme always exhibits better performance than that without using the PWC scheme. It is because the performance of subcarriers with poor SNR conditions (high-frequency ones) will be enhanced, thanks to their corresponding pairing subcarriers with good SNRs (lower-frequency ones). We note that further performance improvement can be achieved by combining the PWC with forward error correction (FEC) code [23]. Thus, with respect to error-free transmission, the users with PWC entail less coding overhead compared with users without PWC due to its significant reduction of maximum BER over subcarrier with PWC. 


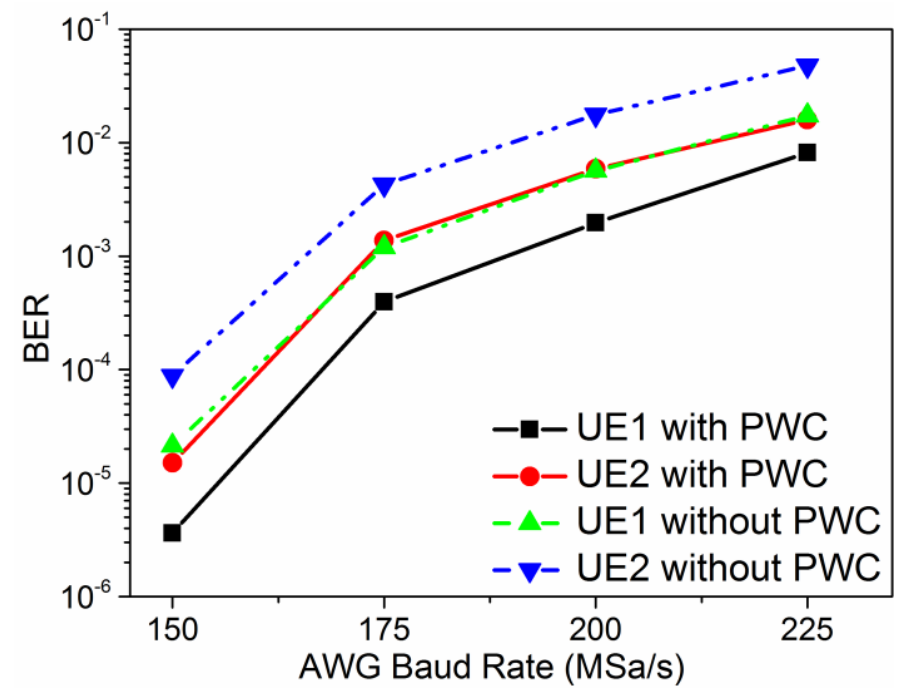

Fig. 8. The BER versus transmitted baud rate for PWC based OFDM-NOMA VLC system.

TABLE I

TOTAL Q ${ }^{2}$ IMPROVEMENT WITH PWC

\begin{tabular}{cc}
\hline \hline AWG Baud Rate $(\mathrm{MSa} / \mathrm{s})$ & $\begin{array}{c}\text { Total } \mathrm{Q}^{2} \text { improvement } \\
\text { with PWC }(\mathrm{dB})\end{array}$ \\
\hline 150 & 1.7 \\
175 & 1.8 \\
200 & 2.7 \\
225 & 3.3 \\
\hline \hline
\end{tabular}

To verify the effectiveness of the proposed PWC-based OFDM-NOMA scheme, we further investigate the system's performance under different AWG baud rates. The performance of the conventional OFDM-NOMA is also investigated for comparison. As shown in Fig. 8, it can be found that the system performance degrades with the increase of transmitted baud rate, due to the limited system bandwidth. For all baud rates, the system with the PWC scheme achieves superior BER performance than the system without the PWC scheme. It is shown in Fig. 8 that with the help of the PWC scheme, the weak user (UE2) can even achieve comparable BER performance with that of the strong user (UE1) in the system without using the PWC scheme. For different AWG baud rate cases, the total $\mathrm{Q}^{2}$ improvements of UE1 and UE2 with PWC are listed in Table I. It can be seen that by using the proposed PWC-based OFDM-NOMA scheme, up to $3.3 \mathrm{~dB}$ $\mathrm{Q}^{2}$ improvement can be achieved when the AWG's baud rate is $225 \mathrm{MSa} / \mathrm{s}$. In addition, it is worth noting that the investigated PWC scheme can also be extended among different users, providing that the extra information of the paired user is available in the PWC decoding stage, which will be extensively investigated in our next study.

\section{CONCLUSION}

In this paper, subcarrier PWC-based OFDM-NOMA is proposed and experimentally demonstrated for VLC. By pairing the low-frequency subcarriers with the high-frequency subcarriers, the high-frequency fading issue of VLC can be effectively resolved. This alleviates the severe error propagation in OFDM-NOMA VLC, thus resulting in significantly improved system performance. Experimental results show that with the optimized power ratio, a total $\mathrm{Q}^{2}$ improvement of up to $3.3 \mathrm{~dB}$ can be achieved by using the proposed scheme. 


\section{ACKNOWLEDGMENTS}

The work was supported in part by the National Natural Science Foundation of China under Grant 61775054, Grant 61377079 and 61571188, in part by the Scientific Research Foundation for the Returned Oversea Chinese Scholars, State Education Ministry of China, in part by Science and Technology Project of Hunan Province (2016GK2011) and in part by HKSAR RGC grant (GRF 14201217).

\section{REFERENCES}

[1] D. Tsonev, S. Videv, and H. Haas, “Towards a $100 \mathrm{~Gb} / \mathrm{s}$ visible light wireless access network," Optics Express, vol. 23, no. 2, p. 1627, Jan. 2015.

[2] A. Jovicic, J. Li, and T. Richardson, "Visible light communication: opportunities, challenges and the path to market," IEEE Communications Magazine, vol. 51, no. 12, pp. 26-32, Dec. 2013.

[3] N. Chi, Y. Zhou, S. Liang, F. Wang, J. Li, and Y. Wang, "Enabling Technologies for High-Speed Visible Light Communication Employing CAP Modulation," Journal of Lightwave Technology, vol. 36, no. 2, pp. 510-518, Jan. 2018.

[4] H. Qian, S. C. Dai, S. Zhao, S. Z. Cai, and H. Zhang, "A Robust CDMA VLC System Against Front-End Nonlinearity," IEEE Photonics Journal, vol. 7, no. 5, pp. 1-9, Oct. 2015.

[5] S. H. Chen and C. W. Chow, "Color-Shift Keying and Code-Division Multiple-Access Transmission for RGB-LED Visible Light Communications Using Mobile Phone Camera," IEEE Photonics Journal, vol. 6, no. 6, pp. 1-6, Dec. 2014.

[6] J. He, F. Long, R. Deng, J. Shi, M. Dai, and L. Chen, "Flexible multiband OFDM ultra-wideband services based on optical frequency combs," IEEE/OSA Journal of Optical Communications and Networking, vol. 9, no. 5, pp. 393-400, May 2017.

[7] Y. Xiao, Z. Wang, J. Cao, R. Deng, Y. Liu, J. He, and L. Chen, "Time-frequency domain encryption with SLM scheme for physical-layer security in an OFDM-PON system," IEEE/OSA Journal of Optical Communications and Networking, vol. 10, no. 1, pp. 46-51, Jan. 2018.

[8] M. Chen, J. He, and L. Chen, "Real-time optical OFDM long-reach PON system over $100 \mathrm{~km} \mathrm{SSMF} \mathrm{using} \mathrm{a} \mathrm{directly}$ modulated DFB laser," IEEE/OSA Journal of Optical Communications and Networking, vol. 6, no. 1, pp. 18-25, Jan. 2014.

[9] Y. Hong, T. Wu, and L. K. Chen, "On the Performance of Adaptive MIMO-OFDM Indoor Visible Light Communications," IEEE Photonics Technology Letters, vol. 28, no. 8, pp. 907-910, Apr. 2016.

[10] X. Huang, S. Chen, Z. Wang, J. Shi, Y. Wang, J. Xiao and N. Chi "2.0-Gb/s Visible Light Link Based on Adaptive Bit Allocation OFDM of a Single Phosphorescent White LED," IEEE Photonics Journal, vol. 7, no. 5, pp. 1-8, Oct. 2015.

[11] R. Deng, J. He, M. Chen, Y. Wei, J. Shi, and L. Chen, "Real-time VLLC-OFDM HD-SDI video transmission system with TS-based SFO estimation," in 2017 Optical Fiber Communications Conference and Exhibition (OFC), 2017, pp. 1-3.

[12] S. M. R. Islam, N. Avazov, O. A. Dobre, and K. s Kwak, "Power-Domain Non-Orthogonal Multiple Access (NOMA) in 5G Systems: Potentials and Challenges," IEEE Communications Surveys Tutorials, vol. 19, no. 2, pp. 721-742, Secondquarter 2017.

[13] F. Lu, M. Xu, L. Cheng, J. Wang, and G.-K. Chang, "Power-Division Non-Orthogonal Multiple Access (NOMA) in Flexible Optical Access With Synchronized Downlink/Asynchronous Uplink," J. Lightwave Technol., JLT, vol. 35, no. 19, pp. 4145-4152, Oct. 2017.

[14] X. Guan, Q. Yang, Y. Hong, and C. C.-K. Chan, "Non-orthogonal multiple access with phase pre-distortion in visible light communication," Opt. Express, OE, vol. 24, no. 22, pp. 25816-25823, Oct. 2016.

[15] B. Lin, W. Ye, X. Tang, and Z. Ghassemlooy, "Experimental demonstration of bidirectional NOMA-OFDMA visible light communications," Opt. Express, OE, vol. 25, no. 4, pp. 4348-4355, Feb. 2017.

[16] J. Shi, Y. Hong, J. He, R. Deng, and L.-K. Chen, "Experimental Demonstration of OQAM-OFDM based MIMO-NOMA over Visible Light Communications," in Optical Fiber Communication Conference (2018), paper M2K.3, 2018, p. M2K.3.

[17] Y. Hong, J. Xu, and L.-K. Chen, "Experimental investigation of multi-band OCT precoding for OFDM-based visible light communications," Optics Express, vol. 25, p. 12908, 2017.

[18] H. Li, Z. Huang, Y. Xiao, S. Zhan, and Y. Ji, "Solution for error propagation in a NOMA-based VLC network: symmetric superposition coding," Optics Express, vol. 25, no. 24, pp.29856-29863, Nov. 2017.

[19] C. Chen, W.-D Zhong, H. Yang, P. Du, and Y. Yang, "Flexible-Rate SIC-Free NOMA for Downlink VLC Based on Constellation Partitioning Coding,” IEEE Wireless Communications Letters, vol. 8, no. 2, pp. 568-571, Apr. 2019.

[20] C. Zhu, B. Song, B. Corcoran, L. Zhuang, and A. J. Lowery, "Improved polarization dependent loss tolerance for polarization multiplexed coherent optical systems by polarization pairwise coding," Opt. Express, vol. 23, no. 21, pp. 27434-27447, Oct. 2015.

[21] B. Song, B. Corcoran, Q. Wang, L. Zhuang, and A. J. Lowery, "Subcarrier Pairwise Coding for Short-Haul L/E-ACO-OFDM," IEEE Photonics Technology Letters, vol. 29, pp. 1584-1587, 2017.

[22] J. He, J. He, and J. Shi, "An Enhanced Adaptive Scheme With Pairwise Coding for OFDM-VLC System," IEEE Photonics Technology Letters, vol. 30, no. 13, pp. 1254-1257, Jul. 2018. 
[23] K. Cushon, P. Larsson-Edefors, and P. Andrekson, "Low-Power 400-Gbps Soft-Decision LDPC FEC for Optical Transport Networks," Journal of Lightwave Technology, vol. 34, no. 18, pp. 4304-4311, Sep. 2016. 